\title{
Optimization of geometrical parameter for V-type alpha Stirling engine based on dimensionless analysis
}

\author{
Varit Senviboon ${ }^{1}$, Banterng Silpsakoolsook ${ }^{2}$, Sutapat Kwankaomeng, ${ }^{1, *}$ \\ ${ }^{1}$ Department of Mechanical Engineering, Faculty of Engineering, King Mongkut's Institute of Technology Ladkrabang, Bangkok, \\ Thailand \\ ${ }^{2}$ Department of Chemistry, Faculty of Science, Siam University, Bangkok, Thailand
}

\begin{abstract}
This paper aims to study the effect of mechanism and geometrical parameters on the shaft work of alpha-type Stirling engine with V-cylinder arrangement and find the appropriate value of geometrical parameters to get the maximum value of shaft work output. The equation of motions of both expansion and compression pistons and the variations of volumes and pressures inside the cylinders are expressed in dimensionless forms. Schmidt analysis and Senft's shaft work theory are used to build dimensionless thermodynamic models in order to derive dimensionless shaft work and optimal geometrical parameters for achieving the maximum efficiency
\end{abstract}

\section{Introduction}

Stirling engine is a heat engine that works as a cycle of expansion and compression of air or other working gas by the effects of temperature differences. From these processes, the conversion of energy from heat to mechanical energy in addition, the Stirling engine is a closed-loop cycle engine that has a constant working gas is sealed without transferring to the environment. In practice, the Stirling engine are classified into three types, based on the configurations and varying displacements of piston and displacer, which are alpha, beta, and gamma. The alpha Stirling engine the simplest pattern form of the Stirling engine.

Karabulut H. [1] built and tested performance of the alpha Stirling engine. The engine provided the maximum power under the heater temperature of $1100{ }^{\circ} \mathrm{C}$ and 2.5 bar of engine's pressurization. Batmaz I, Ustun S [2] employed double heaters for solar power heating V-type Stirling engine which the unpressurized engine gave the best performance at $950{ }^{\circ} \mathrm{C}$ heater surface. A.A. ElEhwany et al. [3] tested and tried to use elbow-bend as heater and cooler part of alpha Stirling engine. The result of the engine power delivers about $13 \%$ more power per cc per $\Delta \mathrm{T}$ than the literature from this previous study. The optimization of the alpha Stirling engine were proposed by Homutescu and Balanescu [4]. The results show that the diameter of the expansion piston must be greater than the compression piston diameter. Lately, Scollo et al. [5] proposed the constructive and redesign of the prototype of the twin cylinder alpha Stirling engine. The engine performance was greatly increased. Recently, Martaj N., Rochelle P. [6] presented a 1D modeling of an alpha double-acting Stirling engine engines and carried out the optimal values of the engine geometry.

According to optimization of the Stirling engine, Senft [7] introduced the concept of effectiveness of mechanism and the relationship between force work and the shaft work of an engine. Later, the same author [8] the Schmidt theory was used to combined and find the optimal values for the phase angle and the swept volume ratio for gamma type Stirling engine. Cheng and Yang [9]. also used this method to evaluate and compare the power of Stirling engine between the alpha, beta, and gamma. The result shows that a beta type has the highest power, followed by the alpha and gamma. But the gamma Stirling engine is the most suitable for low temperature different applications. As the same author [10] the rhombic mechanism variable of the beta Stirling engine is also considered to be integrated with the method, in order to find the optimal mechanism variable and other variable. So that in this paper, propose the optimization by using the slider crank mechanism variable of an alpha Stirling engine to cooperate with this method. All variables considered were phase angle, area ratio between expansion side and compression side, temperature ratio, dead volume ratio and the ratio between connecting rod and crank radius at expansion side and compression side.

\footnotetext{
* Corresponding author: sutapat.kw@kmitl.ac.th
} 


\section{Dimensionless analytical models}

\subsection{Displacement of expansion and compression pistons}

Since the changing of the displacement of expansion, $\bar{y}_{e}$ and compression, $\bar{y}_{c}$ pistons which is driven by slider crank mechanisms affect to the variation of volume inside the engine. The equations of displacement of slider crank mechanisms can be presented in dimensionless form as

$$
\begin{gathered}
\bar{y}_{e}(\theta)=\cos \theta+\sqrt{e_{d}^{2}+(\sin \theta)^{2}} \\
\bar{y}_{c}(\theta)=\cos (\theta-\alpha)+\sqrt{e_{p}^{2}+(\sin (\theta-\alpha))^{2}}
\end{gathered}
$$

Where $e_{d}=l_{e} / r_{e}$ and $e_{p}=l_{c} / r_{c}$. Note that in practical, the variable $e_{p}$ and $e_{d}$ must be more than one. If it isn't, it cannot be a slider crank mechanism.

\subsection{The variations of the volumes inside the cylinders}

The volumes of the expansion side and compression side are changing because of movement of these two pistons in this case are assumed that no dead volume from clearance displacement between cylinder and piston. Let $V_{s e}$ is the swept volume of the expansion side $\left(=A_{e} r_{e} \bar{S}_{e}\right)$ so the equation of volume variation of expansion side, $\bar{V}_{e}$ and compression side, $\bar{V}_{c}$ can be written in dimensionless form as

$$
\begin{aligned}
& \bar{V}_{e}(\theta)=\left[\bar{y}_{e, \text { max }}-\bar{y}_{e}(\theta)\right] / \bar{S}_{e} \\
& \bar{V}_{c}(\theta)=\left[\bar{y}_{c, \text { max }}-\bar{y}_{c}(\theta)\right] / a \bar{S}_{c}
\end{aligned}
$$

Where $a$ is an area ratio that is $a=A_{e} / A_{c}, \bar{V}_{e}(\theta)=$ $V_{e} / V_{s e}, \quad \bar{V}_{c}(\theta)=V_{c} / a V_{s e}$, when $r_{e}=r_{c}$ so that the equation of total working gas volume variation can be written as

$$
\bar{V}_{t}(\theta)=\bar{V}_{h}+\bar{V}_{r}+\bar{V}_{k}+\bar{V}_{e}(\theta)+\bar{V}_{c}(\theta)
$$

At the first three terms are dimensionless volumes of heater $\bar{V}_{h}$, regenerator $\bar{V}_{r}$, and cooler $\bar{V}_{k}$, respectively The first three terms can be written as $\chi=\bar{V}_{h}+\bar{V}_{r}+\bar{V}_{k}$.

\subsection{Pressure and back pressure}

From the Schemidt theory, this theory is used to determine the pressure inside the working space. The temperature at total dead space is estimated as an average of $T_{e}$ and $T_{c}$. The Schemidt theory can be rearranged and presented in dimensionless form as

$$
\begin{aligned}
\bar{p}\left(\theta, \alpha, a, \tau, \chi, e_{p}, e_{d}\right)=p(\theta) / \frac{m R T_{e}}{V_{s e}} \\
=\tau /\left(2 \tau \chi /(1+\tau)+\tau \bar{V}_{e}(\theta)+\bar{V}_{c}(\theta)\right)
\end{aligned}
$$

Where $\tau=T_{c} / T_{e}$ is the temperature ratio, and $\bar{p}$ is dimensionless pressure

As discussed by Senft [7], the optimal value of the back pressure is the average cyclic pressure, which is

$$
\bar{p}_{b}=\sqrt{\bar{p}_{\max } \bar{p}_{\min }}
$$

\subsection{Force, Indicated, and shaft works}

The area under $\bar{p}-\bar{V}_{t}$ diagram is the dimensionless indicated work can be evaluated from $\oint \bar{p} d \bar{V}_{t}$.

Force work is work loss in the mechanisms. For the alpha Stirling engine force work will act at the expansion and compression pistons, the result of the pressure different on both sides of this two pistons. Based on the definition given by Senft [7]. Dimensionless force work can be calculated by

$$
\bar{W}_{f}=\oint\left[\left(\bar{p}-\bar{p}_{b}\right) d \bar{V}_{t}\right]^{-}
$$

Where the symbol [ $]^{-}$means

$$
[n]^{-}=\left\{\begin{array}{r}
-n, n \leq 0 \\
0, n>0
\end{array}\right.
$$

From equation (7) as it is described by Senft [7] can be determine dimensionless shaft work by

$$
\bar{W}_{s}=E \bar{W}_{i}-(1 / E-E) \bar{W}_{f}
$$

Where $E$ is the effectiveness of mechanism, depending on the mechanisms design, friction, speed revolution, mechanism parts manufacturing, lubrication and etc. In this study, value of $E$ is defined to 0.8 .

\section{Numerical Results}

The study effects of mechanism size and parameters were analyzed by theoretical analysis with search method. The results of the parameters are as follows: Fig. 1 shows the effect of temperature ratios on $\bar{W}_{i}, \bar{W}_{s}$ and $\bar{W}_{f}$ at $E=$ $0.8, \chi=0.1, \alpha=90^{\circ}, a=1$ and $e_{p}=e_{d}=5$, then the values of $\bar{W}_{i}$ and $\bar{W}_{s}$ are continuously decreasing, but the value of $\bar{W}_{f}$ increases with the value of $\tau$. From $\tau$ at 0.759 , the value of $\bar{W}_{s}$ is zero. This indicates that the engine cannot work and it implies this engine type is not suitable for use at low temperature differences. The value of $\bar{W}_{i}$ is zero when the value of $\tau=1$, where this point means there is no difference in temperature between hot and cold side. 


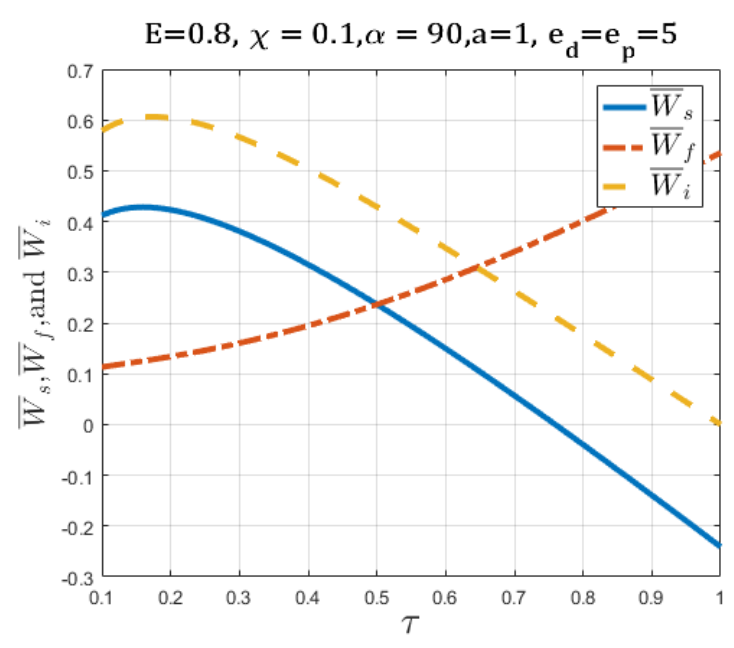

Fig. 1. Effects of temperature ratio on dimensionless works

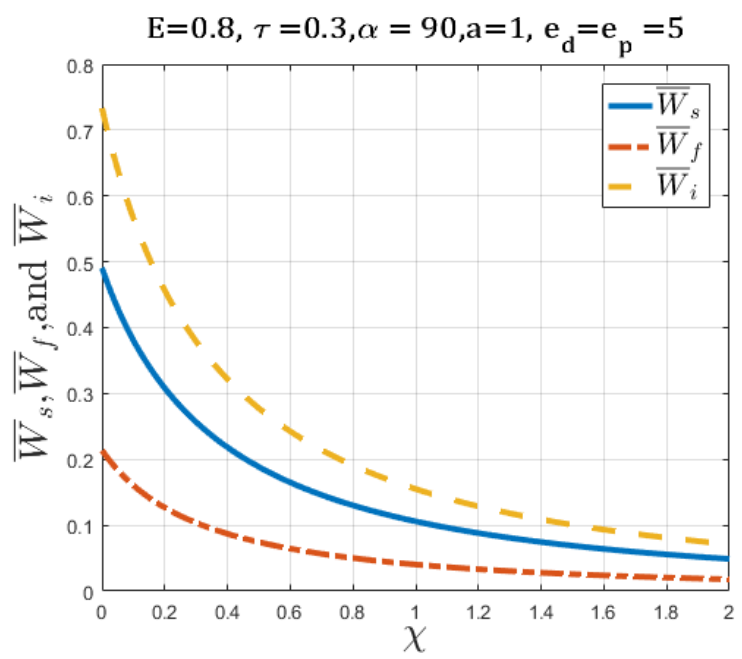

Fig. 2. Effect of total dead volume ratio on dimensionless works

The effect of dead volume ratio $\chi$ on $\bar{W}_{i}, \bar{W}_{s}$ and $\bar{W}_{f}$ is shown in Fig. 2 at $E=0.8, \tau=0.3, \alpha=90^{\circ}, a=1$ and $e_{p}=e_{d}=5$. The results clearly show that the increase in the dead volume ratio will decreases the value of the output. It is observed that the values of $\bar{W}_{i}, \bar{W}_{s}$ and $\bar{W}_{f}$ in the range $\chi$ are between 0 and 0.8 , the value of the output decreases rapidly. But after this, the value starts to drop slowly. In theory, the value of the dead volume ratio should be as low as possible, but in practices, the design and construction of the engine, the heater, regenerator and the cooler part also have dead volume inside. So that this problem cannot be avoided. That why the study of the effect of the dead volume ratio is important.

Fig. 3 show the effect of $e_{p}$ and $e_{d}$ on dimensionless work at $E=0.8, \tau=0.3, \chi=0.1, \alpha=90^{\circ}$ and $a=1$. Let's $e_{p}=e_{d}$. The results of $e_{p}$ and $e_{d}$ are between 1.1 and 5, the value of the dimensionless work is high rate variable. And sooner or later, the work will increase slowly and little by little. As a result, the greater $e_{d}$ and $e_{p}$ will be better transmit work.

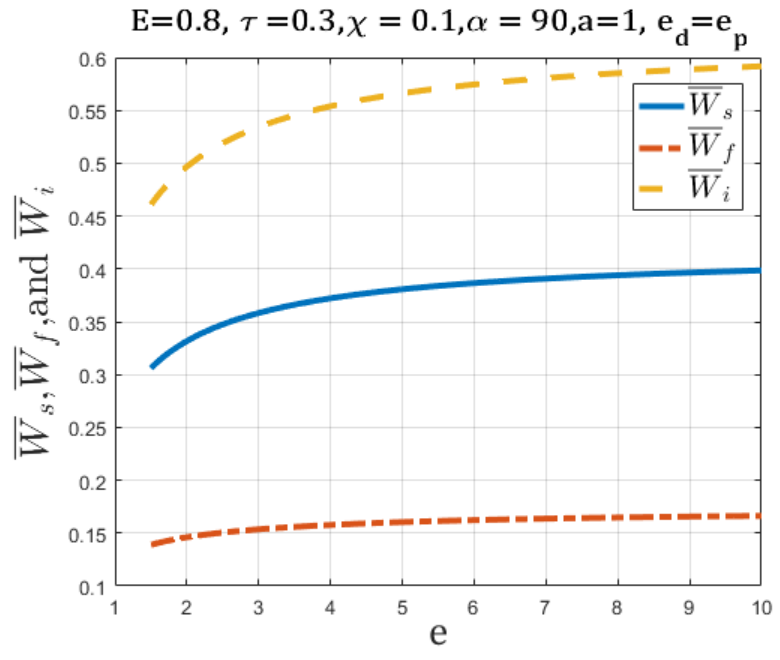

Fig. 3. Effect of $e_{p}$ and $e_{d}$ on dimensionless works

The effect of the phase angle on $\bar{W}_{i}, \bar{W}_{s}$ and $\bar{W}_{f}$ is shown in Fig. 4 at $E=0.8, \tau=0.3, \chi=0.1, a=1$ and $e_{p}=e_{d}=5$. The result is that in the initial phase, the values of $\bar{W}_{i}$ and $\bar{W}_{s}$ are increased to the inverted curve by the maximum $\bar{W}_{s}$ is $\alpha=77^{\circ}$, and then it decreases.

Fig. 5 shows the effect of the area ratio of the expansion on the compression side in case $E=0.8, \tau=$ $0.3, \chi=0.1, \alpha=90^{\circ}$ and $e_{p}=e_{d}=5$.From Fig. 5, it is notice that a must more than 0.22 so the engine can operate. In the range of $a=0.1$ to 1 . It is found that the values of $\bar{W}_{i}$ and $\bar{W}_{s}$ are increasing rapidly, while the value of $\bar{W}_{f}$ decreases rapidly. And then $\bar{W}_{i}$ and $\bar{W}_{s}$ will increase slowly and very little. $\bar{W}_{s}$ have the maximum value of $a=1.79$, and then $\bar{W}_{s}$ will gradually decrease but very little. The result is that the value of the area ratio must be greater than 1, because if the area ratio is less than 1 , then the area of the expansion side smaller than the compression area will be a mechanical disadvantage, resulting in the output power will be decrease or in practices, the engine may not be run.

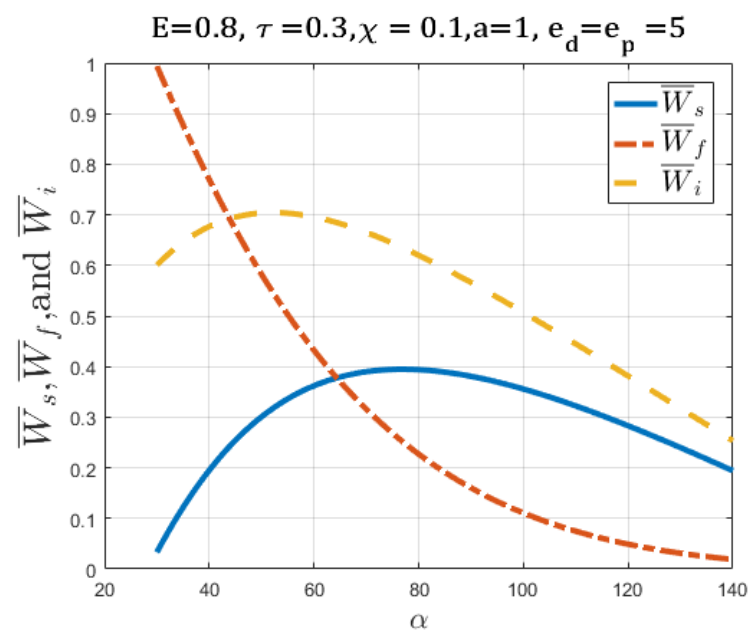

Fig. 4. Effect of phase angle on dimensionless works 


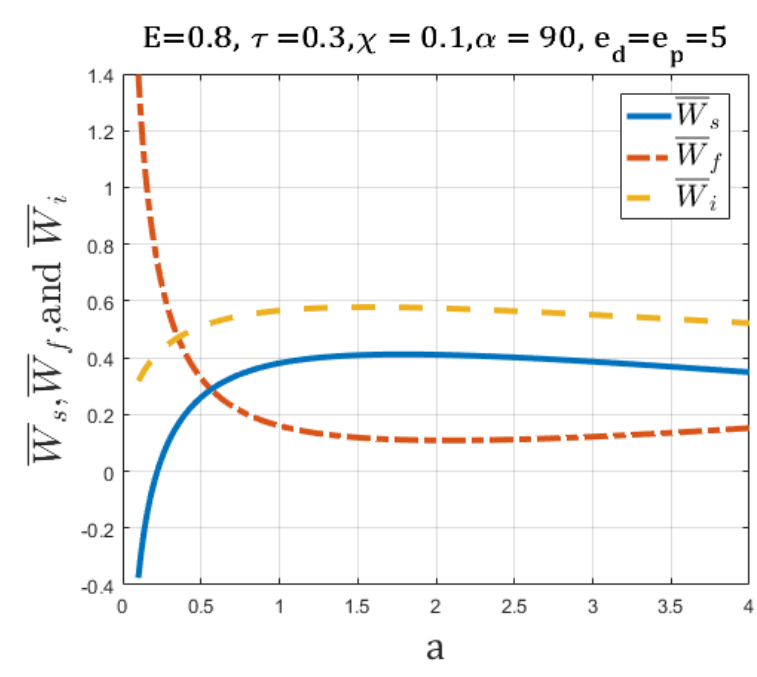

Fig. 5. Effect of area ratio on dimensionless works

The maximum value of the shaft work can be found from two variables that are area ratio and phase angle. So we use these two variables primarily to find the work from the maximum shaft work. Fig. 6 shows contour diagram as the parametric dimensionless shaft work in the function of the area ratio and the phase angle for the alpha V-type Stirling engines at. $E=0.8, \tau=0.3, \chi=0.1$ and $e_{p}=e_{d}=$ 5 . We discover that dimensionless shaft work optimize value are 0.4292 at $a=1.73$ and $\alpha=76^{\circ}$. However the output from variable of area ratio and the phase angle are also depends on the value of effectiveness of mechanism, temperature ratio, dead volume ratio and $e_{p}, e_{d}$. If one value changes, the optimize value will change.

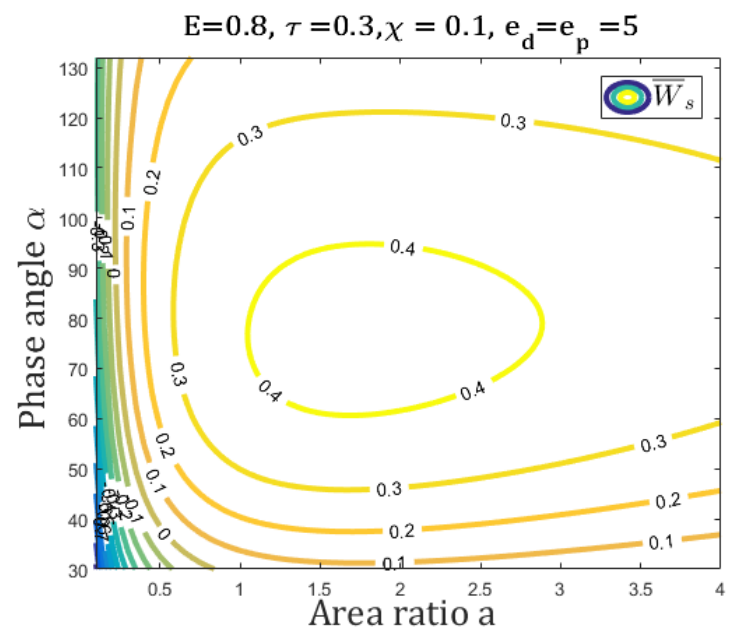

Fig. 6. Contour diagram of dimensionless shaft work as a function of phase angle and area ratio

\section{Conclusion and Remarks}

The result of the assumption at $E=0.8, \chi=0.1, \alpha=$ $90^{\circ}, a=1$ and $e_{p}=e_{d}=5$ indicates that when the temperature ratio increases to 0.759 , the value of dimensionless shaft work $\bar{W}_{s}$ is zero. It's implies that this engine is not suitable for use in low temperature different applications. In addition, the dead volume ratio too much affective to the work of the engine. It is notice that $\bar{W}_{i}, \bar{W}_{S}$ and $\bar{W}_{f}$ are rapidly decreasing as the volume ratio increases. Especially in the range $\chi=0$ to $\chi=0.8$. For the area ratio, it is found that should greater than 0.22 so the engine can operate. At $a=0.1$ to $a=1$ where $\bar{W}_{i}$ and $\bar{W}_{s}$ increase rapidly. It's mean the value of the area ratio must be greater than 1 and the expansion cylinder area must be greater than the compression side.

In terms of the variable of size of the slider crank mechanism, we discover that $e_{d}>1$ and $e_{p}>1$, but the results of these two variables don't have an optimize values. When these two values are higher, the mechanisms are greater transmission power and reduce the effect of side forces on the cylinder wall. Therefore, it is concluded that the variables $e_{p}$ and $e_{d}$ should be designed according to the appropriate proportions. In the other way, when the variable are tried to optimize at. $E=$ $0.8, \tau=0.3, \chi=0.1$ and $e_{p}=e_{d}=5$. The maximum value of dimensionless shaft work is 0.4292 at $a=1.73$ and $\alpha=76^{\circ}$

\section{References}

[1] H. Karabulut, Turk J Engin Environ Sci Manufacturing and Testing of a V-Type Stirling Engine. 24, 71-80 (2000)

[2] I. Batmaz, S. Ustun, Appli. Ener. Design and manufacturing of a $V$-type Stirling engine with double heaters. 85, 1041-1049 (2008).

[3] A.A. El-Ehwany, et al, Ener. Conver. \& Mang. Development of the performance of an alpha-type heat engine by using elbow-bend transposed-fluids heat exchanger as a heater and a cooler. 52, 10101019 (2011)

[4] V M. Homutescu, D-T. Bălănescu, Optimization of diameter Ratio for Alpha-Type Stirling Engine (2010) [Online]. Available at: https://www.agir.ro/buletine/808.pdf [Accessed on 6 March 2018]

[5] L.S. Scollo, et al, Inter. Jour. of Hydro. Ener. Twin cylinder alpha stirling engine combined model and prototype redesign, 38, 1988-1996 (2013)

[6] N. Martaj, P. Rochelle, Inter. Jour. for Sim. of heat eng. 1D modeling of an alpha type Stirling engine, 5: A07 (2014)

[7] JR. Senft, Cambridge Univ Pr, Mechanical efficiency of heat engines, (2007)

[8] JR. Senft, Int. J. Energy Res. Optimum Stirling engine geometry, 26, 1087-1101 (2002)

[9] CH. Cheng, HS. Yang, Appli. Ener. Optimization of geometrical parameters for stirling engine based on theoretical analysis, 92, 395-405 (2012)

[10] CH. Cheng, HS. Yang, Ener. Optimization of rhombic drive mechanism used in beta-type Stirling engine based on dimensionless analysis, 64, 970978 (2013) 